\title{
Neuroprotection of hydroxysafflor yellow A in experimental cerebral ischemia/reperfusion injury via metabolic inhibition of phenylalanine and mitochondrial biogenesis
}

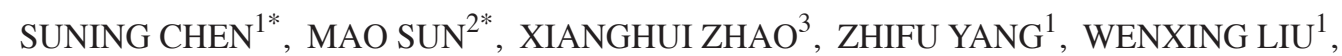 \\ JINYI CAO $^{1}$, YI QIAO ${ }^{1,4}$, XIAOXING LUO ${ }^{5}$ and AIDONG WEN ${ }^{1}$ \\ ${ }^{1}$ Department of Pharmacy, Xijing Hospital, The Fourth Military Medical University; \\ ${ }^{2}$ Department of Biochemistry and Molecular Biology, Center for DNA Typing, The Fourth Military Medical University; \\ ${ }^{3}$ Institute of Neuroscience, Fourth Military Medical University, Xi'an, Shaanxi 710032; \\ ${ }^{4}$ Department of Pharmacology, Jiaotong University School of Medicine, Xi'an, Shaanxi 710049; \\ ${ }^{5}$ Department of Pharmacology, School of Pharmacy, Fourth Military Medical University, Xi'an, Shaanxi 710032, P.R. China
}

Received May 10, 2018; Accepted January 29, 2019

DOI: $10.3892 / \mathrm{mmr} .2019 .9959$

\begin{abstract}
Stroke is the second most frequent cause of mortality, resulting in a huge societal burden worldwide. Timely reperfusion is the most effective therapy; however, it is difficult to prevent ischemia/reperfusion (I/R) injury. In traditional Chinese medicine, hydroxysafflor yellow A (HSYA) has been widely used for the treatment of cerebrovascular disease and as a protective therapy against I/R injury. Evidence has demonstrated that HSYA could reduce the levels of reactive oxygen species and suppress cellular apoptosis; however, whether HSYA alters the metabolic profile as its underlying mechanism for neuroprotection remains unknown. In the present study, using a metabolomic screening, phenylalanine was identified to significantly increase in an experimental model of mouse cerebral I/R injury. Notably, western blotting and $\mathrm{qPCR}$ analysis were conducted to test the expression level of apoptosis-associated factors, and HSYA was identified to be able to protect neuronal cells by reducing phenylalanine level associated with I/R injury. Additionally, these findings were confirmed in primary mouse neurons and PC12 cells
\end{abstract}

Correspondence to: Professor Aidong Wen, Department of Pharmacy, Xijing Hospital, The Fourth Military Medical University, 127 Changle West Road, Xi'an, Shaanxi 710032, P.R. China

E-mail: adwen-2004@hotmail.com

Professor Xiaoxing Luo, Department of Pharmacology, School of Pharmacy, Fourth Military Medical University, 169 Changle West Road, Xi'an, Shaanxi 710032, P.R. China

E-mail:xxluo3@fmmu.edu.cn

"Contributed equally

Key words: hydroxysafflor yellow A, mitochondria biogenesis, neuroprotection, ischemia/reperfusion injury, oxygen and glucose deprivation/reoxygenation, phenylalanine exposed to oxygen and glucose deprivation/reoxygenation (OGD/R) stress. Of note, HSYA was observed to regulate the mRNA expression of key metabolic enzymes, phenylalanine hydroxylase, tyrosine aminotransferase and aspartate aminotransferase, which are responsible for phenylalanine metabolism. Furthermore, by performing mitochondrial labeling and JC-1 fluorescence assay, HSYA was identified to promote mitochondrial function and biogenesis suppressed by OGD/R. The findings of the present study demonstrated that I/R injury could increase the levels of phenylalanine, and HSYA may inhibit phenylalanine synthesis to enhance mitochondrial function and biogenesis for neuroprotection. The present study proposed a novel metabolite biomarker for cerebral I/R injury and the evaluated the efficacy of HSYA as a potential therapeutic treatment I/R injury.

\section{Introduction}

Cerebral stroke remains the most common cause of mortality and a major cause of neurological disability, resulting in notable societal burden worldwide (1). Ischemia restricts the blood supply to certain regions of the brain, resulting in acute glucose and oxygen deprivation (2). Accumulating evidence demonstrated that ischemia/reperfusion (I/R) stress causes oxidative damage and cell apoptosis $(3,4)$. Increasing efforts have been made to determine the mechanism underlying I/R-associated cell stress and injury $(5,6)$; however, whether I/R injury dysregulates the metabolic profile remains unknown.

In traditional Chinese medicine, safflower (Carthamus tinctorius) has been widely used for the treatment of cerebrovascular and cardiovascular diseases (7). The pigments of the Carthamus tinctorius extract contain hydroxysafflor yellow A (HSYA), safflor yellow B, safflomin A, safflomin C and other components (8). As the most important and representative component of safflower, HSYA has a notable therapeutic and protective effects against cerebral and myocardial I/R injury (9). HSYA can inhibit cell apoptosis and reduce the levels of reactive oxygen species (ROS), which helps to rescue 
damaged neurons and promote cell survival (10). Thus, HSYA is widely used for the neuroprotection for cerebral stroke and $\mathrm{I} / \mathrm{R}$ in clinic (11).

The 'Warburg effect' has been widely accepted as a common feature of cancer cells that occurs via metabolic reprogramming (12). Of note, metabolic reprogramming is not only limited to cancer cells but has been reported in disorders of the brain, gut and lungs associated with I/R stress (13-15). In addition, cerebral infarction also caused metabolite alterations and reduced the levels of branched-chain amino acid in ischemic stroke (16). It has been demonstrated that HSYA can activate cell survival signaling via the PI3K/AKT pathway, as well as other pathways (17); however, whether HSYA protects against I/R injury via metabolic alterations requires further investigation.

In the present study, we first identified the metabolic amino acid profile in I/R mouse model. I/R injury notably disturbed the metabolic flux and induced a significant increase in levels of plasma phenylalanine. Notably, treatment with HSYA could recover the alterations in phenylalanine levels induced by I/R stress, and regulate the expression of key enzymes, including phenylalanine hydroxylase (PAH), tyrosine aminotransferase (TAT) and aspartate aminotransferase (GOT1), which are responsible for phenylalanine transformation (18). Notably, HSYA was observed to rescue mitochondrial function and glucose uptake ability. Additionally, HSYA could promote mitochondria biogenesis by increasing the expression of fission-associated dynamin-1-like protein (DRP1), which was downregulated via oxygen and glucose deprivation/reoxygenation (OGD/R) and phenylalanine treatment.

The findings of the present study demonstrated the novel mechanism of I/R injury via upregulating the levels of phenylalanine; HSYA was reported to inhibit increases in phenylalanine levels for neuroprotection via enhancing mitochondrial function and biogenesis. The present study proposed a novel metabolite as a biomarker for cerebral I/R injury and the evaluated the efficacy of HSYA treatment.

\section{Materials and methods}

Mouse model of ischemia and reperfusion. Male C57BL/6 mice (age, 6-8 weeks; weight, 18-20 g) were obtained from the animal center of The Fourth Military Medical University, and housed with a 12 -h light/dark cycle at $18-23^{\circ} \mathrm{C}$, with a humidity of 40-60\% and ad libitum access to food and water. A total of 24 mice were used to generate a model of cerebral I/R. The mice were divided into four groups: Sham operation and I/R, and I/R model treatment groups, which were treated with HSYA ( 5 or $20 \mathrm{mg} / \mathrm{kg}$ ). Transient focal cerebral ischemia was induced by right middle cerebral artery occlusion (MCAO) as described (19). After $2 \mathrm{~h}$, the monofilament was withdrawn to establish the I/R model. Then, two I/R groups of mice were treated with 5 or $20 \mathrm{mg} / \mathrm{kg} /$ day HSYA (intraperitoneal injection) for 3 days. Following treatment and analysis of neurological behavior, $50 \mu 1$ plasma samples were extracted from mouse tail veins, and the mice were subsequently sacrificed. All experiments were approved by The Animal Care Committees of The Fourth Military Medical University (Xi'an, China).
Neurological behavior deficit evaluation. Modified scoring systems for neurological deficits were used to evaluate the neuroprotection effect of HSYA for $72 \mathrm{~h}$ with I/R injury. With slight modifications, a $0-4$ point scale was used to evaluate the neurological deficits observed in all groups (20). The scoring system used was the following: 0 , no deficit; 1 , forelimb flexion; 2 , forelimb flexion and decreased resistance to lateral push; 3 , unidirectional circling; 4, longitudinal spinning, seizure or no movement.

Measurement of infarct zone in the brain. The brain tissues were collected, resected and cut into $2 \mathrm{~mm}$ thick slices using a brain matrix, and treated with 2, 3, 5-triphenyltetrazolium chloride (TTC; Sigma-Aldrich; Merck KGaA, Darmstadt, Germany) at $37^{\circ} \mathrm{C}$ for $30 \mathrm{~min}$ and then fixed in $10 \%$ phosphate-buffered formalin for $45 \mathrm{~min}$ at room temperature. The tissues were imaged with a light microscope (Olympus CX31; Olympus Corporation, Tokyo, Japan; magnification, 4x). The infarction zone was calculated as the percentage of infarction area compared with the ipsilateral hemisphere. The infarct zone was analyzed in five fields of view using MPLAS-500 multimedia color pathological graphic analysis system (Wuhan Qingping Imaging Technology Co., Ltd., Wuhan, China) (21).

Metabolic profiling and quantification. Samples were analyzed using liquid chromatography-tandem mass spectroscopy (MS/MS). A liquid chromatography system (Shimadzu Corporation, Kyoto, Japan) was used to flow the sample into the MS/MS system. A mixture of acetonitrile and water $(70: 30, v / v)$ was used as the carrier solution at a flow rate of $0.14 \mathrm{ml} / \mathrm{min}$. In total, $18 \mu \mathrm{l}$ of the sample was added to the carrier solution; subsequently, the liquid was delivered to the ion source of the MS/MS system without column separation. The MS/MS analysis was conducted with an API 4000+ tandem mass spectrometer (AB Sciex LLC, Framingham, MA, USA) in positive ionization mode and electrospray ionization was performed with nitrogen gas at $400^{\circ} \mathrm{C}$ and the nebulizer pressure was $30 \mathrm{psi}$. In total, two alternating scan modes were defined in the MS setup. Neutral loss scan of m/z 102 was used for neutral amino acids $(25 \mathrm{~V}, 13-17 \mathrm{eV})$. All data acquisition and processing were performed with Analyst software (version 1.5.2; AB Sciex LLC).

Primary culture of mouse cortex neurons. Pregnant female C57BL/6 mice (age, 8-10 weeks; weight, 18-20 g) were obtained from the animal center of The Fourth Military Medical University (Xi'an, China), housed with a 12-h light/dark cycle at $18-23^{\circ} \mathrm{C}$, with a humidity of $40-60 \%$ and ad libitum access to food and water. The mice were sacrificed via $50-70 \% \mathrm{CO}_{2}$ inhalation with a fill rate of $\mathrm{CO}_{2}$ displacement $\sim 20 \%$ of the chamber volume/min. Cerebral cortical neurons from the cerebrum of mice at embryonic age 18.5 (E18.5) were prepared. The cerebral cortex of the brains was dissected out in cold PBS and the tissues were cut into small pieces $\left(1 \mathrm{~mm}^{3}\right)$ and digested with $0.25 \%$ trypsin $+0.04 \%$ EDTA at $37^{\circ} \mathrm{C}$ for $15 \mathrm{~min}$. The cell suspension was centrifuged at $500 \mathrm{x}$ g on $4^{\circ} \mathrm{C}$ for $5 \mathrm{~min}$, and the cell pellet was resuspended in Neurobasal + B27 (Invitrogen; Thermo Fisher Scientific, Inc., Waltham, MA, USA). Following harvest, cells were incubated for 7 days and were subsequently exposed to OGD for $120 \mathrm{~min}$ and treated 
with or without HSYA at a concentration of 1 or $10 \mu \mathrm{M}$ for $20 \mathrm{~h}$ at $37^{\circ} \mathrm{C}$.

OGD/R in primary mouse neurons. OGD was induced as previously described (22). The cells were cultured with glucose-free Dulbecco's modification of Eagle medium (DMEM; Thermo Fisher Scientific, Inc.) under $5 \% \mathrm{CO}_{2}$ and $95 \% \mathrm{~N}_{2}$ at $37^{\circ} \mathrm{C}$ for $120 \mathrm{~min}$. Then, the culture medium was replaced with DMEM, and the sample was incubated in regular conditions, with $5 \% \mathrm{CO}_{2}$ at $37^{\circ} \mathrm{C}$ for $20 \mathrm{~h}$. In all experiments, the $\mathrm{pH}$ of the medium was maintained at 7.2 under OGD conditions.

$O G D / R$ in PC12 cells. Similar to the primary mouse neuronal cells, PC12 cells (American Type Culture Collection, Manassas, VA, USA) were cultured with glucose-free DMEM and infused with $5 \% \mathrm{CO}_{2}$ and $95 \% \mathrm{~N}_{2}$ at $37^{\circ} \mathrm{C}$ for $12 \mathrm{~h}$. Then, the culture medium was replaced with DMEM, and the sample was returned to the regular conditions of $5 \% \mathrm{CO}_{2}$ and $95 \%$ air at $37^{\circ} \mathrm{C}$ for $24 \mathrm{~h}$. In all experiments, the $\mathrm{pH}$ of the medium was maintained at 7.2 under OGD conditions.

Treatment with phenylalanine in PC12 cells. PC12 cells were treated with various concentrations of phenylalanine $(0,0.5$, 1, 2, 4, 8, 16 and 32 mM; Sigma-Aldrich; Merck KGaA) for 48 or $96 \mathrm{~h}$ at $37^{\circ} \mathrm{C}$ prior to MTT assay. The cells were treated with $8 \mathrm{mM}$ phenylalanine for $48 \mathrm{~h}$ in $\mathrm{JC}-1$ prior to western blotting analysis.

Western blot analysis. Radioimmunoprecipitation assay protein extraction buffer was purchased from The Beyotime Institute of Biotechnology (Haimen, China). The total protein was extracted from mouse brain tissues or cultured cells and was subsequently quantified using a bicinchoninic acid assay kit or Bradford's assay kit (Thermo Fisher Scientific, Inc.), respectively. A total of $40 \mu \mathrm{g}$ protein was loaded per lane. Proteins were separated by $12 \%$ SDS-PAGE for 60-80 min. The gel was subsequently transferred on nitrocellulose membranes for $90 \mathrm{~min}$. The membrane was blocked with $5 \%$ fat-free milk for $1 \mathrm{~h}$ at room temperature. Antibodies against phosphorylated protein kinase B (p-Akt; 1:1,000; cat. no. 4058; Cell Signaling Technology, Inc., Danvers, MA, USA), anti-Akt (1:1,000; cat. no. 9272; Cell Signaling Technology, Inc.), anti-cleaved caspase-3 (of c-Casp3; 1:1,000; cat. no. 9664; Cell Signaling Technology, Inc.), anti- $\beta$-catenin (1:1,000; cat. no. 8480; Cell Signaling Technology, Inc.), anti-B-cell lymphoma 2 (BCL2; 1:1,000; cat. no. 2870; Cell Signaling Technology, anti-mitochondrial dynamin like GTPase (OPA1; 1:1,000; cat. no. 67589; Cell Signaling Technology, Inc.), anti-voltage-dependent anion channel (VDAC; 1:1,000; cat. no. 4661; Cell Signaling Technology, Inc.), anti-mitofusin 2 (MFN2; 1:1,000; cat. no. 11925; Cell Signaling Technology, Inc.), anti-fission, mitochondrial 1 (Fis1; 1:1,000, ab71498; Abcam, Cambridge, UK) and anti-neuronal nuclei (NeuN; 1:500; cat. no. 26975-1-AP; ProteinTech Group, Inc., Chicago, IL, USA) were applied overnight at $4^{\circ} \mathrm{C}$. $\beta$-actin (1:2,000; cat. no. AB8227; Abcam) served as the loading control. Secondary antibodies were incubated for $1 \mathrm{~h}$ at room temperature (1:2,000; cat. nos. 7074 and 7076; Cell Signaling Technology, Inc.). Membranes were incubated with chemiluminescent reagents (Pierce; Thermo Fisher Scientific, Inc.) for detecting horseradish peroxidase-labeled antibodies at room temperature prior to x-ray exposure. Western blot analysis was repeated three times, and similar results were obtained. Densitometry was performed using the Image-Pro Plus software (version 6.0; Media Cybernetics, Inc., Rockville, MD, USA).

Reverse transcription-quantitative polymerase chain reaction $(R T-q P C R)$. RNA was extracted using TRIzol ${ }^{\circledR}$ RNA Isolation Reagents (Invitrogen; Thermo Fisher Scientific, Inc.) and cDNA was generated with SuperScript ${ }^{\mathrm{TM}}$ II reverse transcriptase according to the manufacturer's protocol (Invitrogen; Thermo Fisher Scientific, Inc.). A total of 2-5 $\mu \mathrm{g}$ RNA was mixed with reverse transcriptase and incubated at $42^{\circ} \mathrm{C}$ for 50 min to synthesize cDNA. The mRNA levels of mouse brain tissues, primary mouse neuronal cells or PC12 cells exposed to OGD/R were evaluated using a SYBR-Green RT-qPCR kit (Takara Biotechnology Co., Ltd., Dalian, China). qPCR was conducted using a CFX96 Touch $^{\mathrm{TM}}$ RT PCR Detection System (Bio-Rad Laboratories, Inc., Hercules, CA, USA) as follows: Denaturation at $95^{\circ} \mathrm{C}$ for $10 \mathrm{sec}$, primer annealing at $60^{\circ} \mathrm{C}$ for $20 \mathrm{sec}$ and elongation at $72^{\circ} \mathrm{C}$ for $30 \mathrm{sec}$, for 40 cycles. The mouse and rat-specific primers designed are presented in Table I. The relative quantification of each target gene was normalized to GAPDH using the $2^{-\Delta \Delta C q}$ quantification method (23), and the fold change between sham and control group was calculated with three replicates in each group.

Flow cytometry and apoptosis analysis. Cell apoptosis was analyzed using an Annexin V-fluorescein isothiocyanate (FITC) Apoptosis Detection kit (eBioscience; Thermo Fisher Scientific, Inc.) according to the manufacturer's protocols. PC12 or primary mouse neuronal cells at $80 \%$ confluency were harvested using $0.25 \%$ trypsin for $5 \mathrm{~min}$ at $37^{\circ} \mathrm{C}$ and washed twice with PBS. Following centrifugation at $500 \mathrm{x} \mathrm{g}$ for $5 \mathrm{~min}$ at $4^{\circ} \mathrm{C}$, cells were resuspended in solution containing Annexin V-FITC and propidium iodide for $15 \mathrm{~min}$ at room temperature. Subsequently, the cells were analyzed with a flow cytometer and the FACSCanto $^{\text {TM }}$ Plus Software (version 3.0; Becton, Dickinson and Company, Franklin Lakes, NJ, USA).

Dichloro-dihydro-fluorescein diacetate (DCFH-DA) cellular ROS detection. PC12 cells at $80 \%$ confluency were seeded in 6-well plates and stained with $2.5 \mu \mathrm{M}$ DCFH-DA in $1 \mathrm{X}$ dilution buffer provided in the kit (eBioscience; Thermo Fisher Scientific, Inc.) for $20 \mathrm{~min}$ at $37^{\circ} \mathrm{C}$; the cells were washed with $1 \mathrm{X}$ Buffer three times. Subsequently, cells were harvested with $0.25 \%$ trypsin for $5 \mathrm{~min}$ at $37^{\circ} \mathrm{C}$ and washed with PBS twice, followed by analysis with a flow cytometer for the detection of FITC, using the FACSCanto ${ }^{\mathrm{TM}}$ Plus Software (version 3.0; Becton, Dickinson and Company).

JC-1 mitochondrial membrane potential and MitoTracker red assay. PC12 cells were seeded in 6 well plates and labeled according to the manufacturer's protocols (cat. no. ab113850, Abcam). Cells were then cultured for $20 \mathrm{~min}$ at $37^{\circ} \mathrm{C}$ and washed with $1 \mathrm{X}$ dilution buffer provided in the kit (eBioscience; Thermo Fisher Scientific, Inc.) three times. Subsequently, cells were harvested and analyzed with a flow cytometer using the FACSCanto ${ }^{\mathrm{TM}}$ Plus Software 
Table I. Primers for reverse transcription-quantitative polymerase chain reaction.

\begin{tabular}{lll}
\hline Gene name & \multicolumn{1}{c}{ Forward sequence } & \multicolumn{1}{c}{ Reverse sequence } \\
\hline Mouse Pah & 5'-TTGTCCTGGAGAACGGAGTC-3' & 5'-CTGGATTCAATGTGTGTCAGGTT-3' \\
Mouse Gotl & 5'-GCGCCTCCATCAGTCTTTG-3' & 5'-ATTCATCTGTGCGGTACGCTC-3' \\
Mouse Tat & 5'-TGCTGGATGTTCGCGTCAATA-3' & 5'-CGGCTTCACCTTCATGTTGTC-3' \\
Mouse GAPDH & 5'-CTTCACCACCATGGAGGAGGC-3' & 5'-GGCATGGACTGTGGTCATGAG-3' \\
Rat Pah & 5'-ACCCTCTAGGGGTAAATCTTTCA-3' & 5'-GAAGCCCCAATGACACAAGC-3' \\
Rat Got 1 & 5'-TCTGCACGTTGCTTGAGTCT-3' & 5'-GCCGAGAGACAGAGACGATG-3' \\
Rat Tat & 5'-AATGCGGACCTCTGCTATGG-3' & 5'-GACAAGCCATCTCCTGTGCT-3' \\
Rat GAPDH & 5'-GTGAAGCTCATTTCCTGGTATG-3' & 5'-AACTGACGGCCTCTCTCTTG-3'
\end{tabular}

Got1, aspartate aminotransferase; Pah, phenylalanine hydroxylase; Tat, tyrosine aminotransferase.

(version 3.0; Becton, Dickinson and Company). Fluorescence in FL-1 channel and lacking fluorescence in FL-2 channel is considered to indicate mitochondria with depolarized $\Delta \psi$, suggesting apoptosis or dysfunction (24). As for the MitoTracker labelling assay, PC12 cells were incubated with 200 nM MitoTracker Red (Cell Signaling Technology, Inc.; cat. no. 9082) for $20 \mathrm{~min}$ at $37^{\circ} \mathrm{C}$. Following incubation, the cells were rinsed with PBS three times and incubated with regular DMEM culture medium. Subsequently, MitoTracker Red was detected with confocal immunofluorescence analysis using an Olympus FV1000 confocal microscope (Olympus Corporation). In total, five fields per view were imaged (magnification, x400).

2-[N-(7-nitrobenz-2-oxa-1,3-diazol-4-yl)amino]-2-deoxyglucose (2-NBDG) uptake assay. PC12 cells were seeded at $1 \times 10^{5} /$ well in 6 -well plate and cultured for $24 \mathrm{~h}$. Then, the cell culture medium was removed and starved for $2 \mathrm{~h}$ without glucose and serum. The culture medium was replaced with serum with or without $25 \mu \mathrm{M}$ 2-NBDG (Sigma-Aldrich; Merck KGaA), and incubated for $40 \mathrm{~min}$ at $37^{\circ} \mathrm{C}$. Subsequently, the cells were washed with ice-cold PBS three times and collected for analysis with a flow cytometer with settings for applied for FITC detection.

MTT assay. A total of 3,000 cells/well were cultured with $100 \mu \mathrm{l}$ DMEM medium in 96 -well plate at $37^{\circ} \mathrm{C}$ and incubated overnight. Various concentrations of phenylalanine $(0,0.5,1,2,4,8,16$ and $32 \mathrm{mM})$ were applied to the cells for 2 or 4 days at $37^{\circ} \mathrm{C}$. Then, $20 \mu \mathrm{l}$ of $5 \mathrm{mg} / \mathrm{ml} \mathrm{MTT}$ was added to each well and incubated for $4 \mathrm{~h}$ at $37^{\circ} \mathrm{C}$. The medium was removed and $150 \mu \mathrm{l}$ dimethyl sulfoxide was added; the absorbance at $590 \mathrm{~nm}$ was detected with a microplate reader (BioTek Instruments, Inc., Winooski, VT, USA).

Statistical analysis. Data are expressed as the mean \pm standard error. Statistical analysis was performed using a Student's t-test, one-way analysis of variance followed by a Dunnett's post hoc test. $\mathrm{P}<0.05$ was considered to indicate a statistically significant difference. Analyses were performed using Prism (version 7.0; GraphPad Software, Inc., La Jolla, CA, USA). The experiments were performed three times and at least three replicates were present in each group.

\section{Results}

Protective effects of HSYA on neurologic behavior and infarction size with TTC staining. As presented in Fig. 1A, an experimental mouse model of cerebral I/R was generated and the protective effect of HSYA was investigated. After 3 days following $2 \mathrm{~h}$ of I/R treatment, significant increases in neurological behavior deficits in the I/R group compared with the sham group were observed $(\mathrm{P}<0.001)$. Of note, administration of $5 \mathrm{mg} / \mathrm{kg}$ HSYA notably alleviated the neurological deficits in the I/R + HSYA group compared with the I/R group; however, a significant effect was observed in response to $20 \mathrm{mg} / \mathrm{kg}$ HSYA ( $\mathrm{P}<0.01$; Fig. 1B). TTC staining revealed that the infarct area of the $\mathrm{I} / \mathrm{R}$ group was $\sim 37 \%$ of that of the sham group; however, 5 and $20 \mathrm{mg} / \mathrm{kg}$ HSYA significantly decreased the size to 7.2 and $22.4 \%$, respectively $(\mathrm{P}<0.05$; Fig. 1C and D). The results indicated that HSYA had an notable protective effect against cerebral I/R injury. To further confirm the neuroprotective effect of HSYA, western blotting was performed to detect the expression of apoptosis and proliferation-associated proteins. The expression of the proliferation markers p-Akt, $\beta$-catenin and BCL2 were notably decreased and that of c-Casp3 increased following I/R treatment; however, HSYA rescued the expression of p-Akt, $\beta$-catenin and BCL2, and decreased that of c-Casp3 compared with the control (Fig. 1E). In particular, treatment with $20 \mathrm{mg} / \mathrm{kg}$ HSYA significantly increased the expression of neuronal cell marker NeuN (Fig. 1E and F), which indicated the neuroprotective effect of HSYA against I/R.

HSYA regulates the expression of enzymes of phenylalanine metabolism to reprogram the metabolic amino acid profile associated with cerebral I/R. To investigate whether I/R and HSYA alter the metabolic profile, plasma samples from the sham and I/R groups treated with or without HSYA were extracted from mouse tail veins and centrifuged at $10,500 \mathrm{xg}$ at room temperature for $5 \mathrm{~min}$ for metabolic screening. The results revealed a significant increase in the levels of plasma phenylalanine (from $56.69 \pm 1.097$ to $95.55 \pm 13.01 \mu \mathrm{mol} / \mathrm{l}$ ) with I/R stress compared with the sham group Treatment with HSYA significantly reduced the levels of phenylalanine $(\mathrm{P}<0.05$; Fig. 2A). The data suggested that HSYA could 

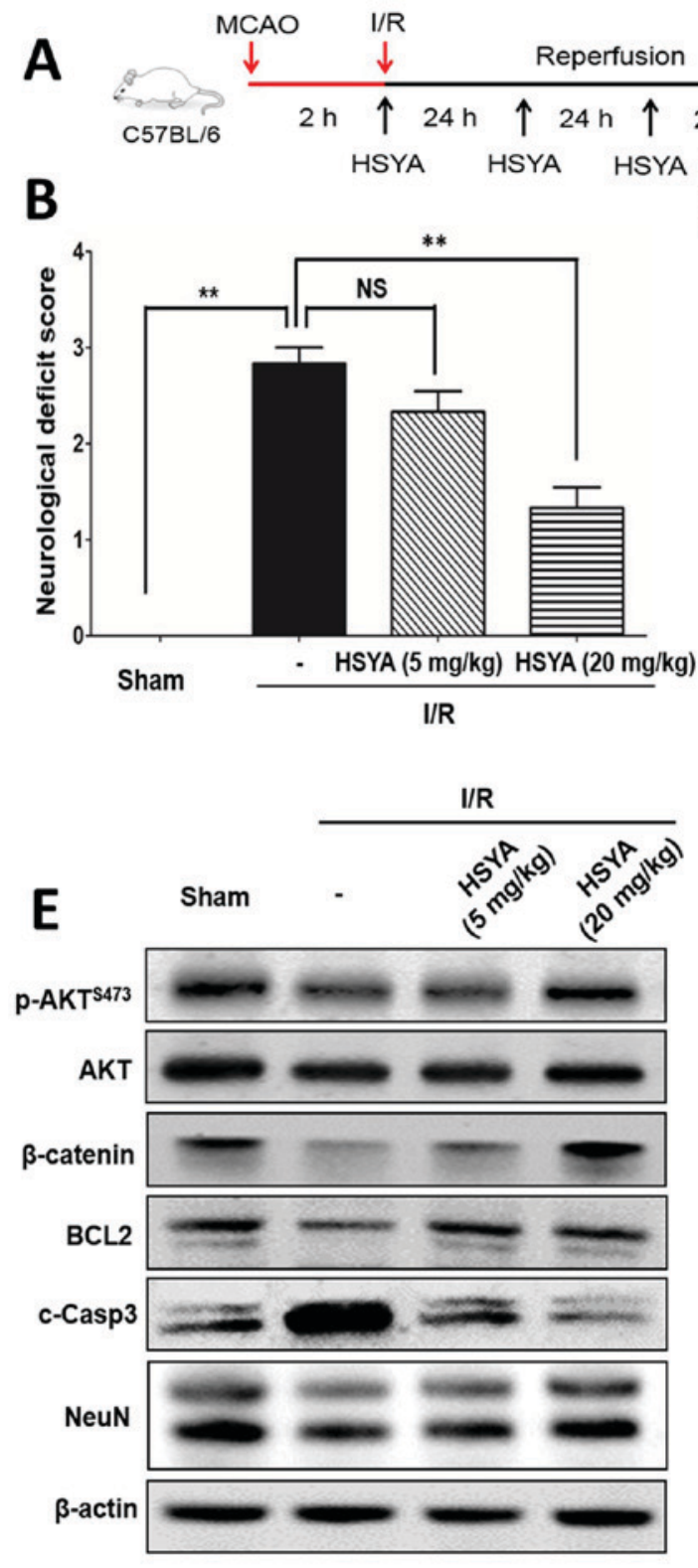

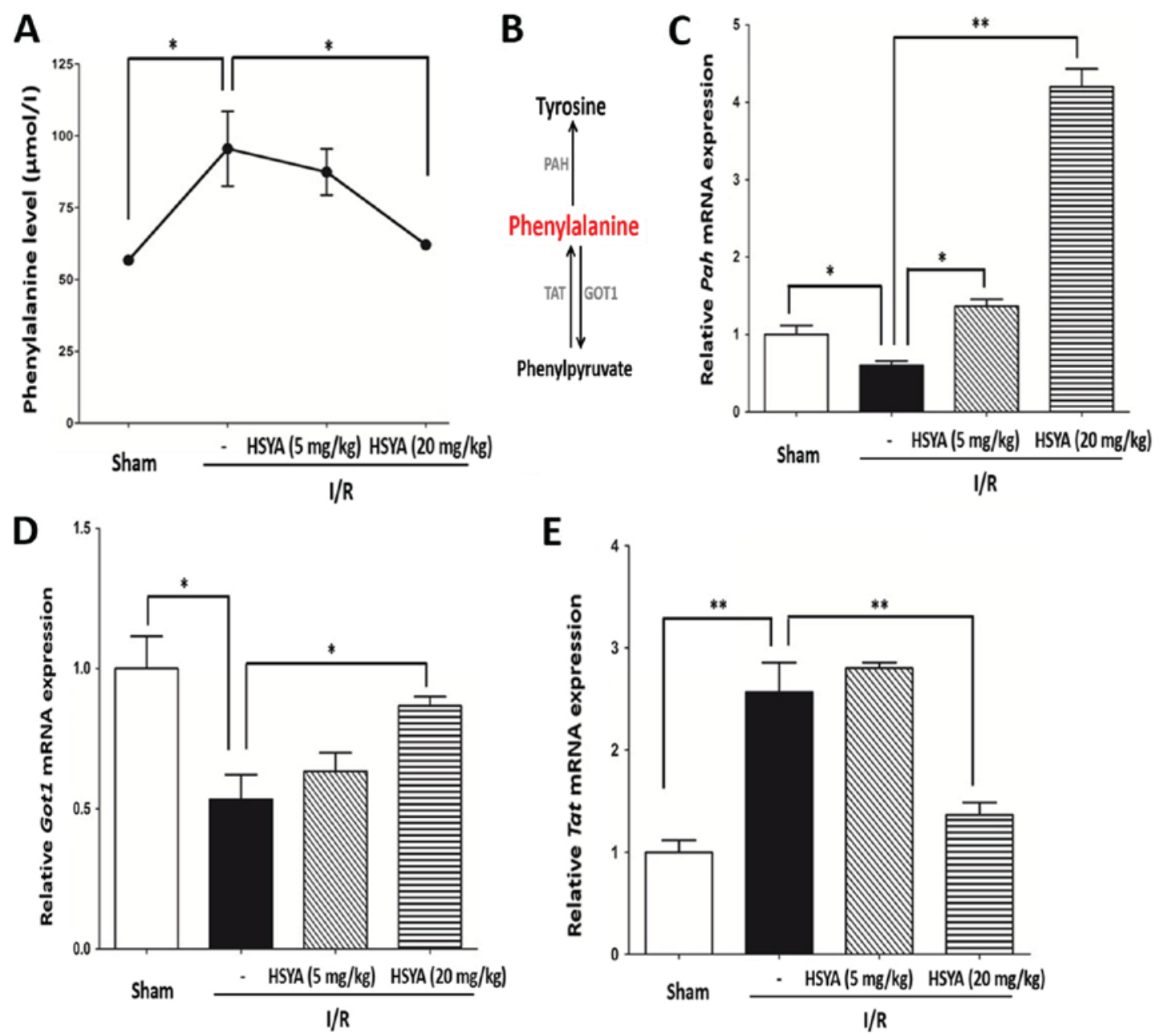

Figure 2. HSYA regulates the levels of phenylalanine and the metabolic enzymes expression caused by cerebral I/R in vivo. (A) I/R stress increased the plasma levels of phenylalanine, which was reversed by HSYA. (B) Transformation flux of phenylalanine. Phenylalanine can be metabolized to phenylpyruvate and tyrosine via GOT1 and TAT, and PAH, respectively. HSYA reduced the levels of phenylalanine by regulating the expression of key metabolic enzymes. mRNA expression and relative quantification of (C) Pah, (D) Gotl and (E) Tat. The data are expressed as the mean \pm standard error of the mean. "P<0.05, ${ }^{* *} \mathrm{P}<0.01$. GOT1, aspartate aminotransferase; HYSA, hydroxysafflor yellow A; I/R, ischemia/reperfusion; PAH, phenylalanine hydroxylase; TAT, tyrosine aminotransferase.

demonstrated that HSYA significantly inhibited the expression of c-casp3; the expression of p-Akt, BCL2 and NeuN had recovered in response to HSYA following I/R (Fig. 3B). Additionally, as expected, HSYA could significantly inhibit neuronal cell apoptosis induced by OGD/R treatment; the effect was notably greater in response to $10 \mu \mathrm{M}$ HSYA than $1 \mu \mathrm{M}$ (Fig. 3C and D). Therefore, HSYA may protect mouse neuronal cells from OGD/R stress.

To evaluate whether HSYA also alters the metabolic profile associated with OGD/R in primary neurons, metabolic screening was performed. The results revealed alterations in phenylalanine levels, which was similar to the in vivo model (I/R injury). HSYA also restricted the increase of phenylalanine in primary neurons with OGD/R. Thus, alterations in the levels of phenylalanine were markedly coincident in vivo and in vitro (Figs. $2 \mathrm{~A}$ and $3 \mathrm{E}$ ). Furthermore, the mRNA expression levels of Pah, Got 1 and Tat in the mouse neuron model was similar to the results of the in vivo model (Fig. 3F-H). Therefore, HSYA may recover the levels of phenylalanine by regulating the expression of key metabolic enzymes in a mouse model of $\mathrm{I} / \mathrm{R}$ and in primary mouse neuronal cells exposed to
OGD/R stress. Thus, the neuroprotective properties of HSYA may occur by recovering the levels of phenylalanine dysregulated by $\mathrm{I} / \mathrm{R}$ injury.

HSYA protects $P C 12$ cells exposed to OGD/R stress via the regulation of phenylalanine metabolism. In addition, to further confirm the neuroprotective effects of HSYA, as previously reported (25), an OGD/R model in PC12 cells was generated (Fig. 4A). Similar to the primary mouse neuronal cells, OGD/R significantly induced the apoptosis of PC12 cells compared with the control; HSYA significantly protected against apoptosis caused by OGD/R stress $(\mathrm{P}<0.01$; Fig. 4B). Of note, the levels of phenylalanine in $\mathrm{PC} 12$ cells were significantly increased in response to $\mathrm{OGD} / \mathrm{R}$ compared with the control, whereas the levels were significantly reduced following treatment with HSYA ( $\mathrm{P}<0.05$; Fig. 4C).

Furthermore, alterations in the mRNA expression levels of Pah, Gotl and Tat in PC12 cells were similar within the in vivo model and primary mouse neuronal cells (Fig. 4D-F). Therefore, in vivo, mouse I/R injury and neuronal cells 
A

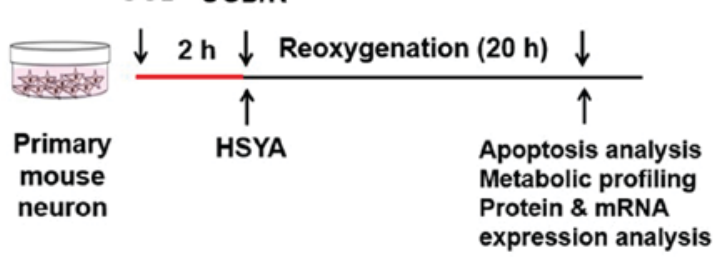

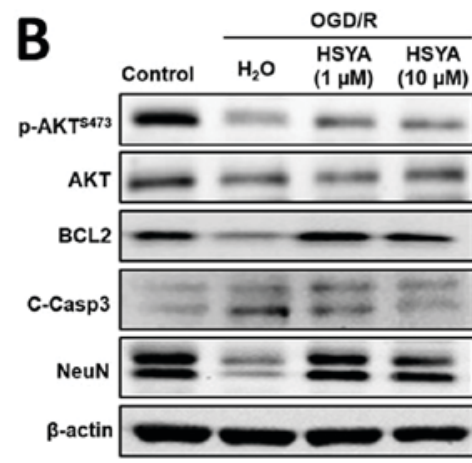

E
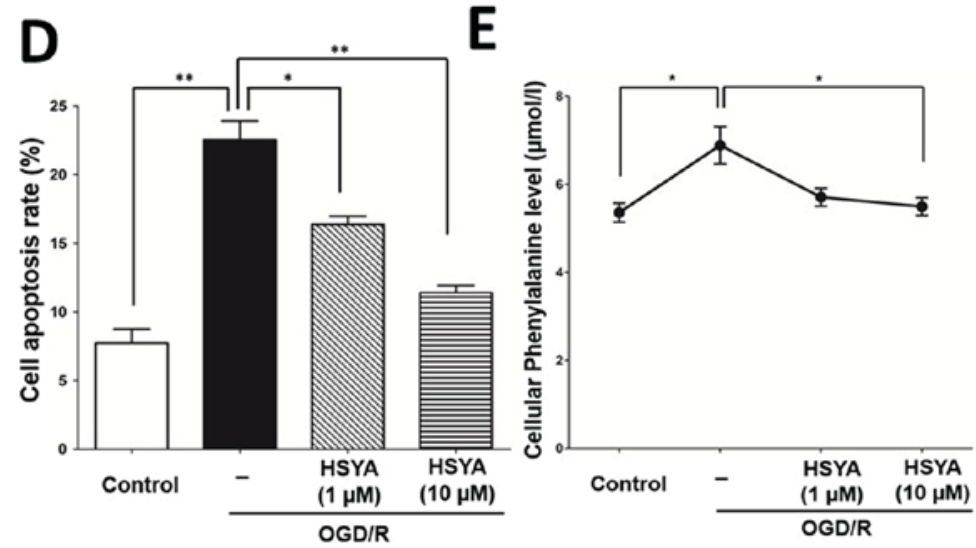

H

G

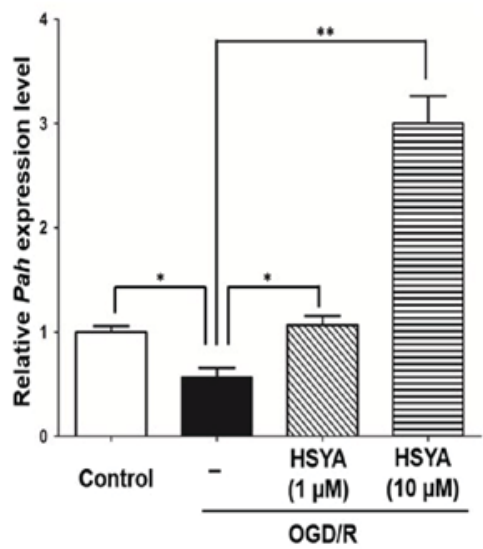

H

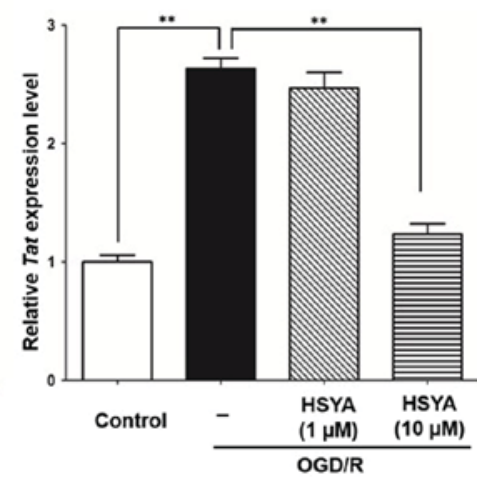

Figure 3. HSYA regulates the levels of phenylalanine in primary mouse neuronal cells with OGD/R stress. (A) Experimental scheme for primary mouse neurons with OGD/R treatment. (B) Analysis of p-Akt, BCL2, cleaved caspase-3 and NeuN levels by means of western blotting in primary mouse neuron with OGD/R treatment. (C) Analysis of apoptosis via flow cytometry. (D) Quantification of apoptosis. (E) Quantification of phenylalanine levels in the control and OGD/R groups treated with or without HSYA. HSYA regulates the expression of key metabolic enzymes in primary mouse neuronal cells. mRNA expression and relative quantification of $(\mathrm{F})$ Pah, $(\mathrm{G})$ Got1 and $(\mathrm{H})$ Tat. The data are expressed as the mean \pm standard error of the mean. ${ }^{*} \mathrm{P}<0.05,{ }^{* *} \mathrm{P}<0.01$. Akt, protein kinase B; BCL2, B-cell lymphoma 2; c-Casp3, cleaved caspase 3; GOT1, aspartate aminotransferase; HYSA, hydroxysafflor yellow A; PAH, phenylalanine hydroxylase; NeuN, neuronal nuclei; OGD/R, oxygen and glucose deprivation/reoxygenation; p, phosphorylated; TAT, tyrosine aminotransferase.

in vitro exposed to $\mathrm{OGD} / \mathrm{R}$ stress exhibited increased levels of phenylalanine by suppressing the expression of key metabolic enzymes, pah and Gotl, and upregulating Tat expression (Fig. 4G). HSYA may reduce the levels of phenylalanine by regulating the expression of these three key enzymes (Fig. 4G). Thus, the neuroprotective properties of HSYA may occur by recovering the levels of phenylalanine induced by $\mathrm{I} / \mathrm{R}$ injury and OGD/R stress (Fig. 4G).

HSYA promotes mitochondrial function and biogenesis associated with its neuroprotective effect against $O G D / R$ stress in PC12 cells. To clarify the neuroprotective mechanism of HSYA, whether HSYA affected the mitochondrial function in PC12 cells was investigated. As presented in Fig. 5A, OGD/R stress significantly increased the ROS levels of PC12 cells compared with the control, as detected by DCFH-DA analysis. As expected, HSYA significantly reduced the ROS levels induced by OGD/R compared with the OGD/R group $(\mathrm{P}<0.01$; Fig. 5A). JC-1 staining revealed that HSYA $(10 \mu \mathrm{M})$ significantly promoted mitochondrial function by increasing membrane potential suppressed by $\mathrm{OGD} / \mathrm{R}$ stress $(\mathrm{P}<0.01$; Fig. 5B). Additionally, HSYA significantly enhanced the 


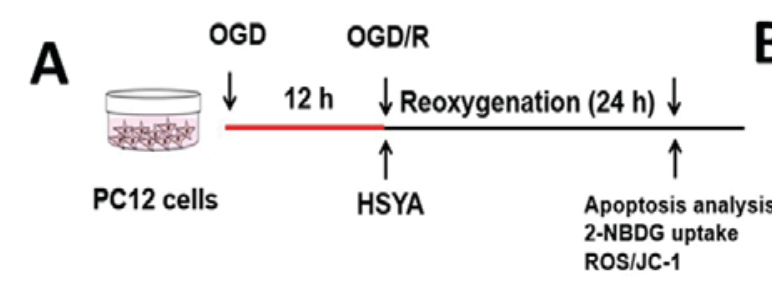

B
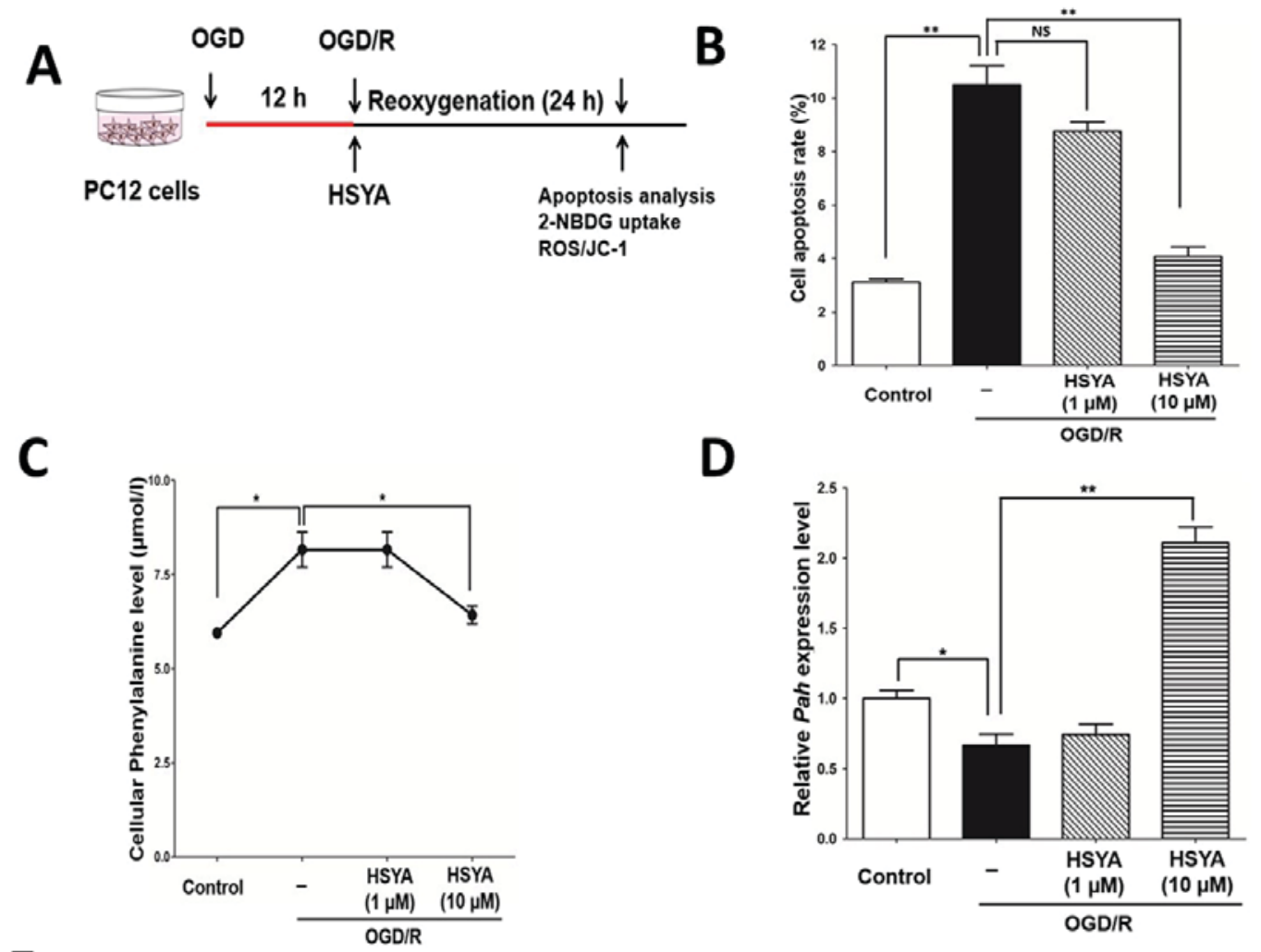

E
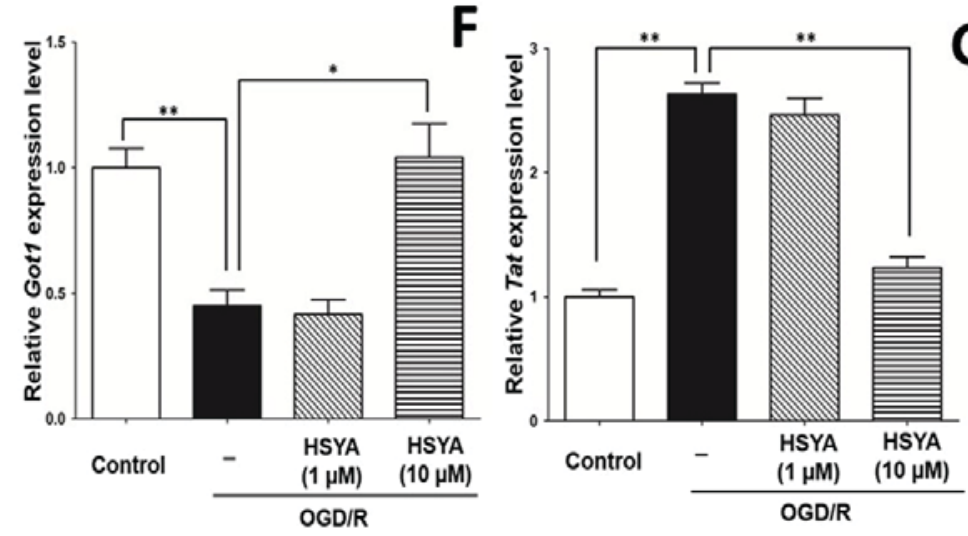

G

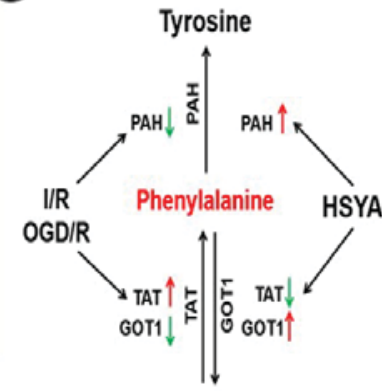

Phenylpyruvate

Figure 4. HSYA suppresses the apoptosis and alters the expression of enzymes associated with the metabolism of phenylalanine in PC12 cells with OGD/R stress. (A) Experimental scheme for PC12 cells exposed to OGD/R. (B) Apoptosis analysis and quantification in each group via flow cytometry. (C) Quantification of phenylalanine levels in the control and OGD/R groups treated with or without HSYA. HSYA regulated the expression of key metabolic enzymes of phenylalanine in PC12 cells. Relative quantification of the mRNA expression of (D) Pah, (E) Gotl and (F) Tat. (G) Summarization of HSYA regulating metabolic enzymes expression to reduce the levels of phenylalanine. All data are expressed as the mean \pm standard error of the mean. ${ }^{*} \mathrm{P}<0.05,{ }^{* *} \mathrm{P}<0.01$. Got1, aspartate aminotransferase; HYSA, hydroxysafflor yellow A; Pah, phenylalanine hydroxylase; OGD/R, oxygen and glucose deprivation/reoxygenation; p, phosphorylated; Tat, tyrosine aminotransferase.

glucose uptake ability of PC12 cell as determined via 2-NBDG analysis ( $\mathrm{P}<0.01$; Fig. 5C).

Furthermore, via MitoTracker red assay, it was demonstrated that mitochondria were small and rod-like following OGD/R (Fig. 5D); however, HSYA promoted the morphological alteration of mitochondria to a narrow and elongated shape, indicating mitochondria fission and biogenesis potential (26). Most importantly, HSYA treatment significantly increased the expression of mitochondria fission protein DRP1 and notably inhibited that of the mitochondria fusion protein OPA1 compared with the OGD/R group (Fig. 5E and F). Additionally, treatment with HSYA increased the protein expression level of
VDAC, a mitochondrial marker, suggesting an increase in mitochondrial number (Fig. 5E). These data demonstrated HSYA could promote mitochondria function and biogenesis for its neuroprotective effects in PC12 cells exposed to OGD/R stress.

HSYA recovers the function of mitochondria suppressed by phenylalanine in PC12 cells. As an endogenous metabolite, it is difficult to inhibit the production of phenylalanine. To evaluate whether phenylalanine can directly impair mitochondrial function in neuronal cells, the proliferation of PC12 cells treated with phenylalanine was analyzed. As presented in Fig. 6A, higher concentrations of phenylalanine inhibited 

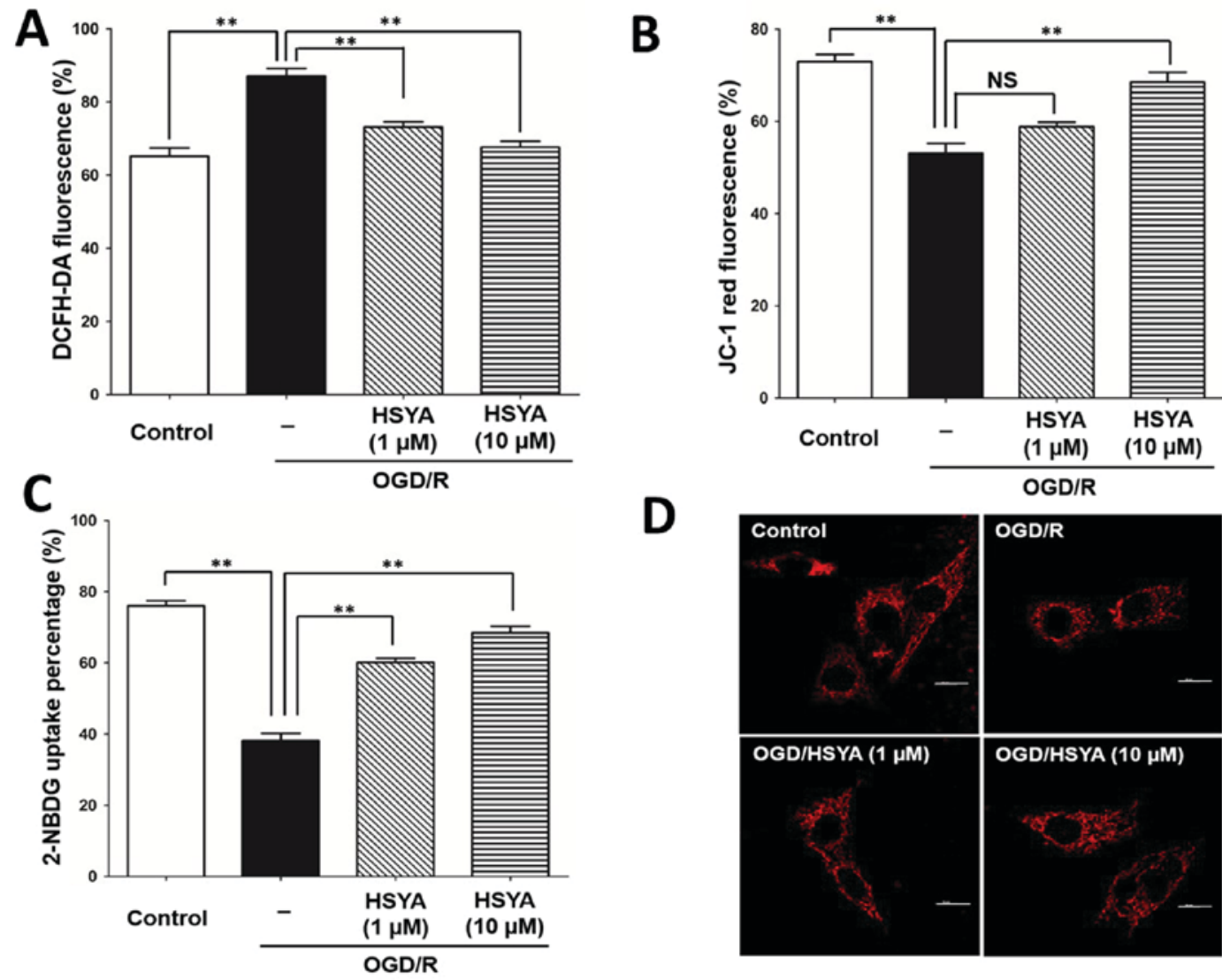

D
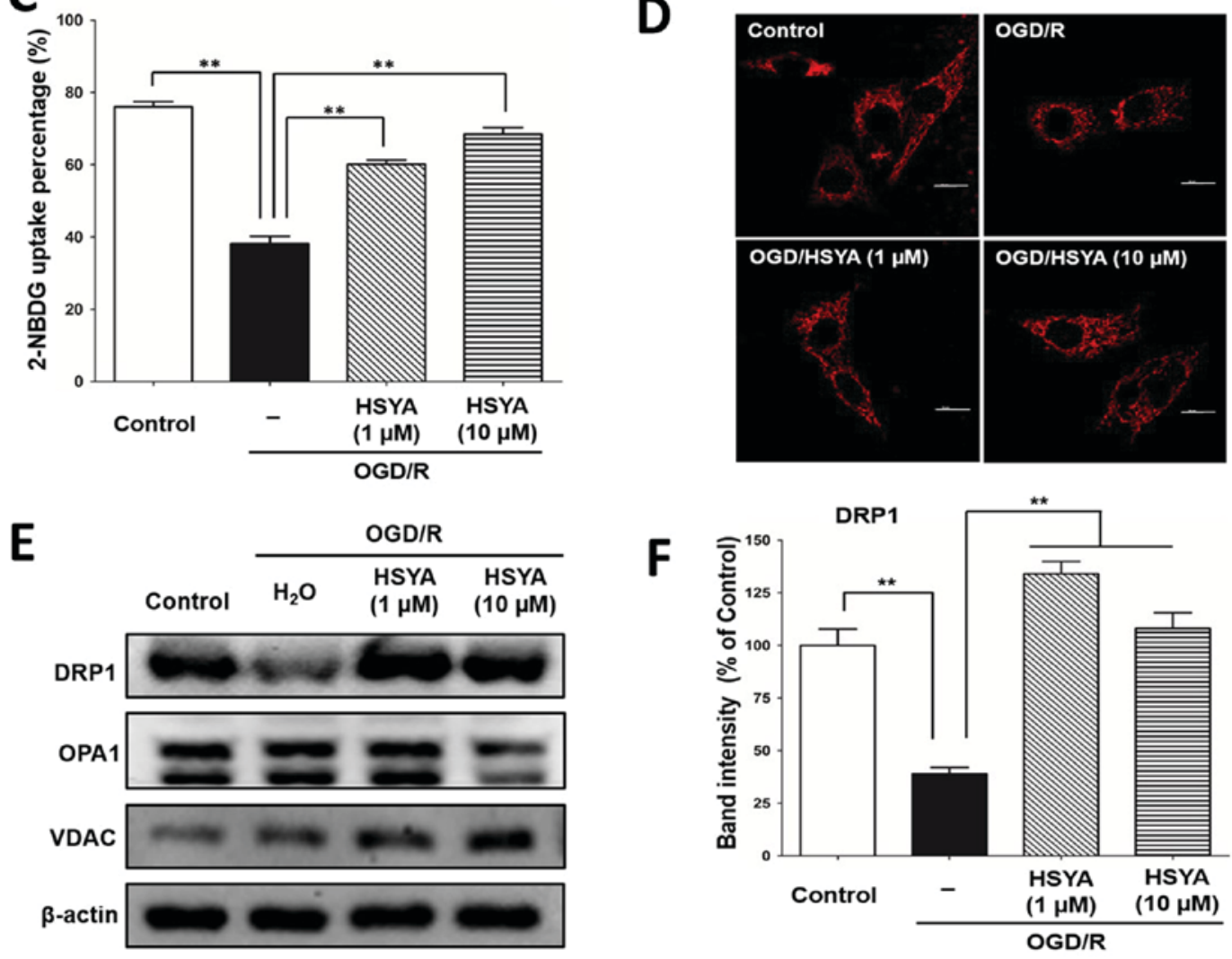

Figure 5. HSYA promotes mitochondrial function and biogenesis in PC12 cells exposed to OGD/R stress. (A) DCFH-DA analysis in PC12 cells with OGD/R stress and HSYA treatment as indicated. (B) JC-1 red fluorescence assay in PC12 cells with OGD/R stress and HSYA treatment. (C) Glucose uptake ability in PC12 cells exposed to OGD/R stress and HSYA as detected by 2-NBDG labelling. (D) MitoTracker Red fluorescence in PC12 cells was detected with a confocal immunofluorescence microscope. Mitochondrial shape was compared in each group. Scale bar=50 $\mu \mathrm{m}$. (E) Western blotting analysis of mitochondrial fission protein DRP1, fusion protein OPA1 and mitochondrial marker VDAC. $\beta$-actin was the internal control. (F) Band intensity quantification of DRP1 in (E). All data are expressed as the mean \pm standard error of the mean. ${ }^{* *} \mathrm{P}<0.01 .2$-NBDG, 2-[N-(7-nitrobenz-2-oxa-1,3-diazol-4-yl)amino]-2-deoxyglucose; DCFH-DA, dichloro-dihydro-fluorescein diacetate; DRP1, dynamin-1-like protein; HYSA, hydroxysafflor yellow A; OPA1, dynamin-like GTPase; NS, not significant; VDAC, voltage-dependent anion-selective channel 1; OGD/R, oxygen and glucose deprivation/reoxygenation.

the proliferation of PC12 cells (16 and $32 \mathrm{mM})$. Analysis of $\mathrm{JC}-1$ red fluorescence demonstrated that phenylalanine significantly inhibited the mitochondrial function in PC12 cells, and HSYA $(10 \mu \mathrm{M})$ was able to increased mitochondrial function impaired upon treatment with phenylalanine $(\mathrm{P}<0.05$; Fig. 6B). In addition, phenylalanine significantly reduced the expression of the mitochondrial fission protein DRP1 expression compared with the control, which was rescued by HSYA treatment (Fig. 6C and D). Treatment with phenylalanine did not alter the protein expression level of Fis1. However, the protein expression level of MFN2 was slightly increased upon treatment with phenylalanine, although the mechanism underlying MFN2 upregulation remains to be investigated. The findings suggested that HSYA may promote mitochondrial function and biogenesis suppressed by phenylalanine in PC12 cells.

\section{Discussion}

Stroke is the second most common cause of mortality worldwide, causing $\sim 6.2$ million cases of mortality annually (27). There are two major types of stroke: Ischemic and hemorrhagic. 
A

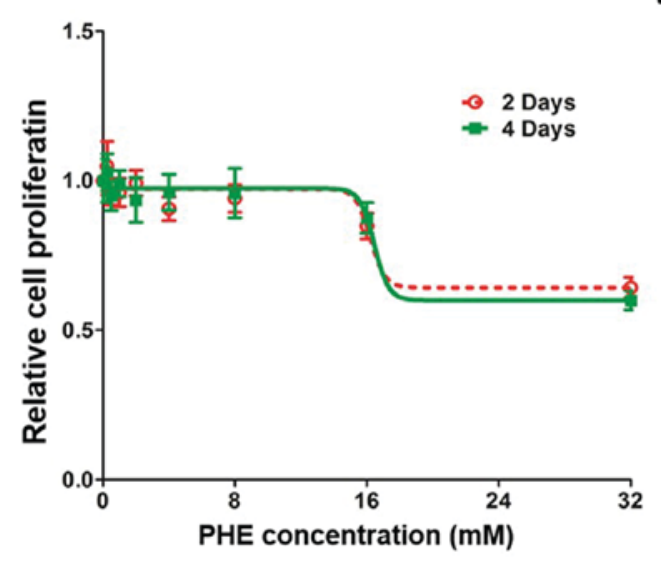

C

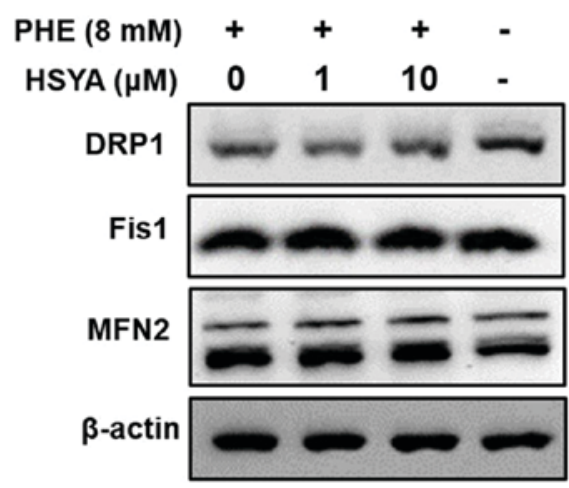

B

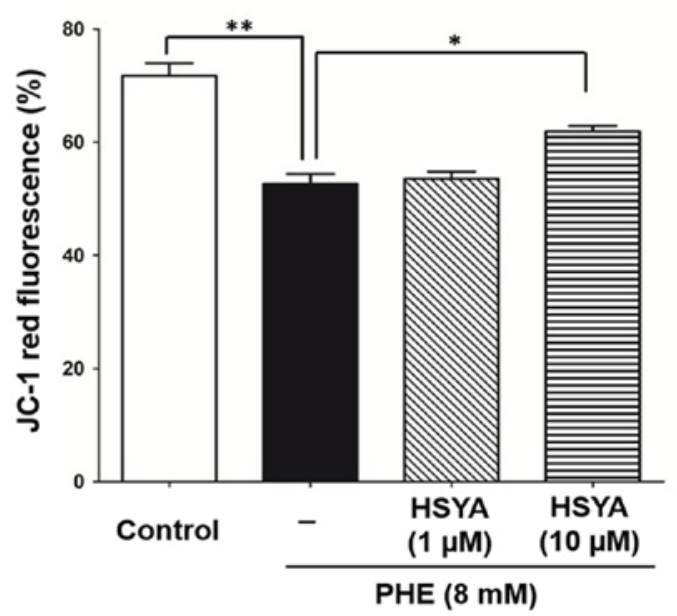

D

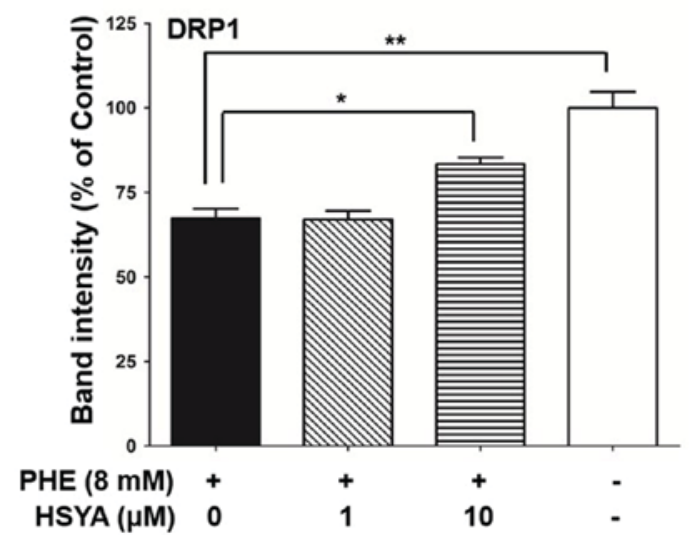

Figure 6. HSYA protects mitochondrial function suppressed by PHE in PC12 cells. (A) MTT assay in PC12 cells with PHE treatment for 2 and 4 days with increased concentration $(0,0.5,1,2,4,8,16$ and $32 \mathrm{mM})$. (B) JC-1 red fluorescence assay in PC12 cells with $8 \mathrm{mM}$ PHE and HSYA (1 and $10 \mu \mathrm{M})$ treatment as indicated. (C) Western blotting analysis of mitochondrial fission protein DRP1 and Fis1, and fusion protein MFN2. $\beta$-actin was the internal control. (D) Band intensity quantification of DRP1. All data are expressed as the mean \pm standard error of the mean. ${ }^{*} \mathrm{P}<0.05,{ }^{* *} \mathrm{P}<0.01$. DRP1, dynamin-1-like protein; Fis1, mitochondrial fission 1 protein; HYSA, hydroxysafflor yellow A; MFN2, mitofusin-2; OGD/R, oxygen and glucose deprivation/reoxygenation; PHE, phenylalanine.

Ischemic stroke, which occurs in $\sim 87 \%$ of patients, is mainly caused by an interruption of the blood supply to certain regions of the brain (28). Despite major advances in stroke imaging and treatment, stroke continues to threaten patients and causes familial and societal burden. The initial goal of therapy in ischemic stroke is to restore blood flow. The phenomenon that successful alleviation of regional tissue hypoxia exacerbates reperfusion injury in the form of cell death requires further investigation. Numerous studies using cerebral I/R animal models have identified the mechanism of $I / R$ injury and provided a wide array of neuroprotective strategies to reduce the deleterious effects of reperfusion injury (3); however, the approaches for predicting the efficacy of these strategies are limited.

Several studies reported that the levels of numerous metabolites were altered with cerebral ischemia (16). In particular, branched chain amino acids are notably reduced in ischemic stroke, and the extent correlates with poor neurological outcome (29). In the present study, alterations in the metabolic amino acid profile due to cerebral I/R injury were observed. $\mathrm{I} / \mathrm{R}$ stress increased the levels of phenylalanine and altered metabolic flux. The findings of the present study demonstrated that $\mathrm{I} / \mathrm{R}$ and $\mathrm{OGD} / \mathrm{R}$ stress caused a significant increase in phenylalanine. Furthermore, the significant alterations in the mRNA expression levels of the key metabolic enzymes Pah, Tat and Gotl were reported, which are responsible for the metabolism of phenylalanine (18). The underlying mechanism as to how $\mathrm{I} / \mathrm{R}$ stress regulates the expression of metabolic enzymes remains unknown; however, the findings of the present study indicates that the increased ROS levels induced by I/R injury may be involved.

HSYA has been widely used for the treatment of cerebrovascular diseases in clinical practice. Our previous study revealed that HSYA could reduce ROS levels and activate cellular survival signaling in a myocardial I/R rat model (30). Additionally, the neuroprotective effect of HSYA in a cerebral I/R mouse model was demonstrated in the present study. Furthermore, HSYA reduced the levels of malondialdehyde, and increased those of glutathione and SOD to suppress ROS; thus, apoptosis was inhibited (data not shown). The present study also proposed that HSYA could activate Akt and $\beta$-catenin signaling to promote neuronal cell survival. 


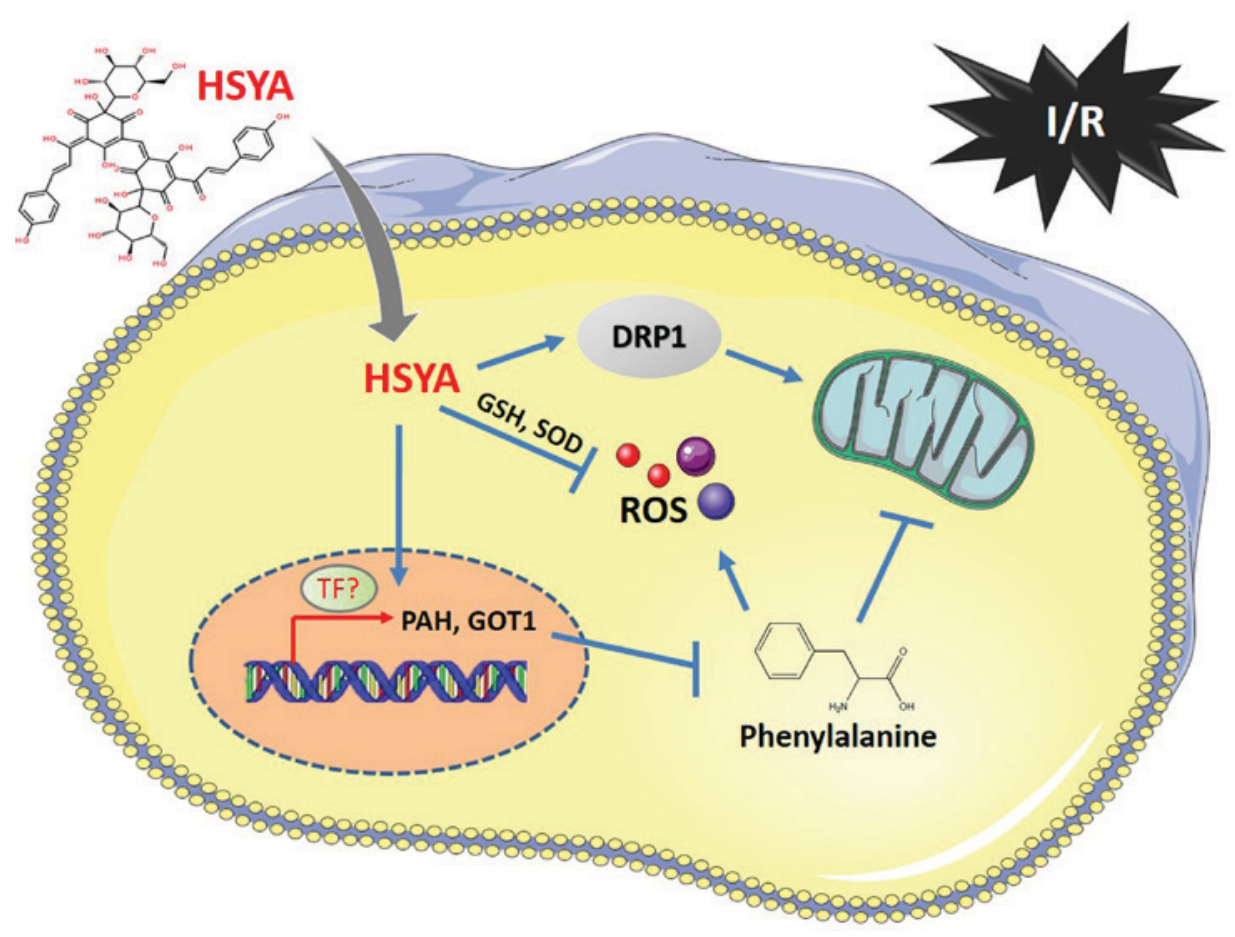

Figure 7. Diagram of HSYA regulating the levels of phenylalanine, mitochondrial function and biogenesis for neuroprotection. I/R injury caused increased ROS and phenylalanine levels to induce neuronal cell apoptosis. HSYA could reduce the production of ROS and phenylalanine by regulating the expression of key metabolic enzymes, including PAH, TAT and GOT1. In addition, HSYA could promote mitochondrial function and biogenesis by upregulating mitochondria fission protein DRP1. GOT1, aspartate aminotransferase; GSH, glutathione; HYSA, hydroxysafflor yellow A; PAH, phenylalanine hydroxylase; ROS, reactive oxygen species; SOD, superoxide dismutase.

Metabolic screening was conducted to identify alterations in the metabolic amino acid profile in the present study. The results revealed that increases in the levels of phenylalanine were induced by cerebral I/R injury. Interestingly, the in vivo and in vitro models demonstrated that HSYA could reduce alterations in phenylalanine levels caused by $\mathrm{I} / \mathrm{R}$ and $\mathrm{OGD} / \mathrm{R}$ stress. Furthermore, we explored the potential mechanism and reported that HSYA could regulate the expression of the key enzymes Pah, Tat and Gotl, which are responsible for phenylalanine transformation. Finally, whether phenylalanine can directly impair the function of mitochondria in neuronal cells was determined. Thus, the levels of phenylalanine may be an ideal biomarker for evaluating HSYA as a therapeutic agent.

As a metabolite, phenylalanine possesses a physiological function associated with amino acid metabolism. A previous study demonstrated that phenylalanine may inhibit neurite outgrowth by interfering with L1-mediated cell adhesion (31). The results of the present study revealed that higher concentrations of phenylalanine inhibited the function of mitochondria and cell proliferation; however, phenylalanine alone could not induce apoptosis. Additional conditions, including an increased concentration of phenylalanine or a combination of multiple metabolites, may lead to an increase in apoptosis.

Mitochondria are critical for cellular metabolism and the regulation of apoptosis. The biogenesis of mitochondria is mostly dependent on the process of mitochondrial fission and fusion (32). In the present study, I/R and OGD/R stress increased the levels of phenylalanine, damaged mitochondrial function and induced ROS production (Fig. 7). Whereas, HSYA could reduce phenylalanine levels and promote mitochondrial function via the upregulation of mitochondrial fission protein DRP1. Thus,
HSYA may promote mitochondria function and biogenesis in association with its neuroprotective effects. The present study proposed a novel metabolite as a biomarker for cerebral I/R injury and provided a novel mechanism for the development of therapeutic strategies based on HSYA to treat ischemic strokes.

\section{Acknowledgements}

The authors would like to thank Professor Xiaoqiang Li (Department of Pharmacology, School of Pharmacy, Fourth Military Medical University, Xi'an, China) for providing advice on the experimental design and critical comments on the present manuscript.

\section{Funding}

The present study was supported by The National Natural Science Foundation of China (grant nos. 81573549 and 81503280).

\section{Availability of data and materials}

The datasets used and/or analyzed during the current study are available from the corresponding author on reasonable request.

\section{Authors' contributions}

$\mathrm{AW}$ and XL designed and supervised the experiments. SC and MS conducted the mouse I/R injury, OGD/R model, metabolomics analysis, qPCR, western blotting and the cellular experiments. XZ performed the experiments on primary 
neuron cells. ZY, WL and JC performed the cellular reactive oxygen species detection analysis and the cell proliferation assay. YQ performed the statistical analysis. ZY, WL, JC and YQ gave critical comments and revised the manuscript. All authors read and approved the final manuscript.

\section{Ethics approval and consent to participate}

The present study was approved by the Animal Care Committees of the Fourth Military Medical University (Xi'an, China).

\section{Patient consent for publication}

Not applicable.

\section{Competing interests}

The authors declare that they have no competing interests.

\section{References}

1. Chu SF, Zhang Z, Zhang W, Zhang MJ, Gao Y, Han N, Zuo W, Huang $\mathrm{HY}$ and Chen NH: Upregulating the expression of Survivin-HBXIP complex contributes to the protective role of IMM-H004 in transient global cerebral Ischemia/reperfusion. Mol Neurobiol 54: 524-540, 2017.

2. Sarami Foroshani M, Sobhani ZS, Mohammadi MT and Aryafar M: Fullerenol nanoparticles decrease blood-brain barrier interruption and brain edema during cerebral ischemia-reperfusion injury probably by reduction of interleukin- 6 and matrix metalloproteinase-9 transcription. J Stroke Cerebrovasc Dis 27: 3053-3065, 2018.

3. Wiklund L, Patnaik R, Sharma A, Miclescu A and Sharma HS: Cerebral tissue oxidative ischemia-reperfusion injury in connection with experimental cardiac arrest and cardiopulmonary resuscitation: Effect of mild hypothermia and methylene blue. Mol Neurobiol 55: 115-121, 2018.

4. Gong L, Tang Y, An R, Lin M, Chen L and Du J: RTN1-C mediates cerebral ischemia/reperfusion injury via ER stress and mitochondria-associated apoptosis pathways. Cell Death Dis 8: e3080, 2017.

5. Li Y, Wang M and Wang S: Effect of inhibiting mitochondrial fission on energy metabolism in rat hippocampal neurons during ischemia/reperfusion injury. Neurol Res: 1-8, 2016 (Epub ahead of print).

6. Guo J, Yong Y, Aa J, Cao B, Sun R, Yu X, Huang J, Yang N, Yan L, Li X, et al: Compound danshen dripping pills modulate the perturbed energy metabolism in a rat model of acute myocardial ischemia. Sci Rep 6: 37919, 2016

7. Jiang S, Shi Z, Li C, Ma C, Bai X and Wang C: Hydroxysafflor yellow $\mathrm{A}$ attenuates ischemia/reperfusion-induced liver injury by suppressing macrophage activation. Int $\mathbf{J}$ Clin Exp Pathol 7 : 2595-2608, 2014.

8. Bai Y, Lu P, Han C, Yu C, Chen M, He F, Yi D and Wu L: Hydroxysafflor yellow A (HSYA) from flowers of Carthamus tinctorius $\mathrm{L}$. and its vasodilatation effects on pulmonary artery. Molecules 17: 14918-14927, 2012.

9. Sun L, Yang L, Xu YW, Liang H, Han J, Zhao RJ and Cheng Y: Neuroprotection of hydroxysafflor yellow $A$ in the transient focal ischemia: Inhibition of protein oxidation/nitration, 12/15-lipoxygenase and blood-brain barrier disruption. Brain Res 1473: 227-235, 2012.

10. Wang X, Ma Z, Fu Z, Gao S, Yang L, Jin Y, Sun H, Wang C, Fan W, Chen L, et al: Hydroxysafflor Yellow A protects neurons from excitotoxic death through Inhibition of NMDARs. ASN Neuro 8: pii: 2016.

11. Li CY, Yin JG, Zhang J, Wang XX, Xu MJ, Liu F, Zou JD and Ju WZ: Pharmacokinetic profiles of hydroxysafflor yellow A following intravenous administration of its pure preparations in healthy Chinese volunteers. J Ethnopharmacol 162: 225-230, 2015.

12. Svensson RU and Shaw RJ: Cancer metabolism: Tumour friend or foe. Nature 485: 590-591, 2012.
13. Zhao Q, Wu J, Hua Q, Lin Z, Ye L, Zhang W, Wu G, Du J, Xia J, $\mathrm{Chu} \mathrm{M}$ and $\mathrm{Hu} \mathrm{X}$ : Resolvin D1 mitigates energy metabolism disorder after ischemia-reperfusion of the rat lung. J Transl Med 14: 81, 2016.

14. Hoehn RS, Seitz AP, Jernigan PL, Gulbins E and Edwards MJ: Ischemia/Reperfusion injury alters sphingolipid metabolism in the gut. Cell Physiol Biochem 39: 1262-1270, 2016.

15. Gellert L, Knapp L, Nemeth K, Herédi J, Varga D, Oláh G, Kocsis K, Menyhárt A, Kis Z, Farkas T, et al: Post-ischemic treatment with L-kynurenine sulfate exacerbates neuronal damage after transient middle cerebral artery occlusion. Neuroscience 247: 95-101, 2013.

16. Kimberly WT, Wang Y, Pham L, Furie KL and Gerszten RE: Metabolite profiling identifies a branched chain amino acid signature in acute cardioembolic stroke. Stroke 44: 1389-1395, 2013.

17. Song Y, Long L, Zhang N and Liu Y: Inhibitory effects of hydroxysafflor yellow A on PDGF-BB-induced proliferation and migration of vascular smooth muscle cells via mediating Akt signaling. Mol Med Rep 10: 1555-1560, 2014

18. Rodriguez B, Torres N, Rincon AR, Bourges H, Panduro A and Tovar AR: Hepatic phenylalanine-hydroxylase and tyrosine-aminotransferase mRNA levels in rats adapted to diets with different concentrations of protein. Rev Invest Clin 48: 413-419, 1996.

19. Zhang J,LiF, Liu X, Shen L, Liu J, Su J,Zhang W, Deng Y, Wang L, Liu N, et al: The repression of human differentiation-related gene NDRG2 expression by Myc via Miz-1-dependent interaction with the NDRG2 core promoter. J Biol Chem 281: 39159-39168, 2006.

20. Ganan-Gomez I, Wei Y, Yang H, Boyano-Adanez MC and Garcia-Manero G: Oncogenic functions of the transcription factor Nrf2. Free Radic Biol Med 65: 750-764, 2013.

21. Wu Y, Wang L, Dai C, Ma G, Zhang Y, Zhang X and Wu Z: Neuroprotection by platelet-activating factor acetylhydrolase in a mouse model of transient cerebral ischemia. Neurosci Lett 558: 26-30, 2014.

22. Hu Z, Yang B, Mo X and Zhou F: HspB8 mediates neuroprotection against $\mathrm{OGD} / \mathrm{R}$ in N2A cells through the phosphoinositide 3-kinase/Akt pathway. Brain Res 1644: 15-21, 2016.

23. Livak KJ and Schmittgen TD: Analysis of relative gene expression data using real-time quantitative PCR and the 2(-Delta Delta C(T)) method. Methods 25: 402-408, 2001.

24. Chazotte B: Labeling mitochondria with JC-1. Cold Spring Harbor Protoc 2011: pii: pdb.prot065490, 2011

25. Liu L, Huang W, Wang J, Song H, Cen J and Ji B: Anthraquinone derivative exerted hormetic effect on the apoptosis in oxygen-glucose deprivation-induced $\mathrm{PC} 12$ cells via ERK and Akt activated Nrf2/HO-1 signaling pathway. Chem Biol Interact 262: 1-11, 2017.

26. Yang JL, Mukda S and Chen SD: Diverse roles of mitochondria in ischemic stroke. Redox Biol 16: 263-275, 2018.

27. Winterholler M, Hollander C, Kerling F, Weber I, Dittrich S, Türk M and Schröder R: Stroke in duchenne muscular dystrophy: A retrospective longitudinal study in 54 patients. Stroke 47: 2123-2126, 2016.

28. Morris NA, Merkler AE, Gialdini G and Kamel H: Timing of incident stroke risk after cervical artery dissection presenting without ischemia. Stroke 48: 551-555, 2017.

29. Liu Y, Luo F, Xu Y, Wang B, Zhao Y, Xu W, Shi L, Lu X and Liu Q: Epithelial-mesenchymal transition and cancer stem cells, mediated by a long non-coding RNA, HOTAIR, are involved in cell malignant transformation induced by cigarette smoke extract. Toxicol Appl Pharmacol 282: 9-19, 2015.

30. Wei G, Yin Y, Duan J, Guo C, Zhu Y, Wang Y, Xi M and Wen A: Hydroxysafflor yellow A promotes neovascularization and cardiac function recovery through HO-1/VEGF-A/SDF-1 $\alpha$ cascade. Biomed Pharmacother 88: 409-420, 2017.

31. Hartwig C, Gal A, Santer R, Ullrich K, Finckh U and Kreienkamp HJ: Elevated phenylalanine levels interfere with neurite outgrowth stimulated by the neuronal cell adhesion molecule L1 in vitro. FEBS Lett 580: 3489-3492, 2006.

32. Bockler S, Chelius X, Hock N, Klecker T, Wolter M, Weiss M, Braun RJ and Westermann B: Fusion, fission, and transport control asymmetric inheritance of mitochondria and protein aggregates. J Cell Biol 216: 2481-2498, 2017.

This work is licensed under a Creative Commons Attribution 4.0 International (CC BY-NC 4.0) License 